\title{
REVIEW ARTICLE \\ Face masks to prevent transmission of influenza virus: a systematic review
}

\author{
B. J. COWLING ${ }^{1 *}$, Y. ZHOU ${ }^{1}$, D. K. M. IP ${ }^{1}$, G. M. LEUNG ${ }^{1}$ AND A. E. AIELLO \\ ${ }^{1}$ School of Public Health, The University of Hong Kong, Hong Kong Special Administrative Region, China \\ ${ }^{2}$ Department of Epidemiology, Center for Social Epidemiology \& Population Health, School of Public Health, \\ University of Michigan, Ann Arbor, MI, USA
}

(Accepted 16 December 2009; first published online 22 January 2010)

\section{SUMMARY}

Influenza viruses circulate around the world every year. From time to time new strains emerge and cause global pandemics. Many national and international health agencies recommended the use of face masks during the 2009 influenza A (H1N1) pandemic. We reviewed the English-language literature on this subject to inform public health preparedness. There is some evidence to support the wearing of masks or respirators during illness to protect others, and public health emphasis on mask wearing during illness may help to reduce influenza virus transmission. There are fewer data to support the use of masks or respirators to prevent becoming infected. Further studies in controlled settings and studies of natural infections in healthcare and community settings are required to better define the effectiveness of face masks and respirators in preventing influenza virus transmission.

Key words: Infectious disease control, infectious disease epidemiology, influenza, public health.

\section{INTRODUCTION}

Pandemic influenza A (H1N1) virus emerged in Mexico in early 2009 and rapidly spread worldwide. Severity of illness now appears to be more moderate than initially feared $[1,2]$, although high population attack rates would be associated with significant numbers of severe infections, hospitalizations and deaths. While some governments, particularly in the developed world, have large antiviral stockpiles on hand and contracts for vaccines that are now in production, the primary interventions currently available in both developed and less-developed settings are nonpharmaceutical [3, 4]. At the population level, these

\footnotetext{
* Author for correspondence: Dr B. J. Cowling, School of Public Health, The University of Hong Kong, Units 624-7, Cyberport 3, Pokfulam, Hong Kong. (Email: bcowling@hku.hk)
}

can include border controls to delay cross-border transmission, and social distancing measures such as school or workplace closures. At the individual level, interventions to reduce transmission include improved hygiene and the use of face masks, respirators, and other physical barriers [5]. We conducted a systematic review [6] to investigate the evidence supporting the effectiveness of face masks in reducing influenza virus infection under controlled and natural conditions.

\section{METHODS}

\section{Search strategy}

On 18 August 2009 we searched the following databases for articles published in English from January 1960 to August 2009: PubMed (1960-2009), Science Citation Index (Web of Science) (1970-2009), and the 
Cochrane Library (1988-2009). We searched for articles using the following search strategy:

\#1: 'facemask' OR 'facemasks' OR 'mask' OR 'masks' OR 'respirator' OR 'respirators' OR 'N100' OR 'N99' OR 'N95' OR 'P2' OR 'FFP2'

\#2: 'influenza' OR 'flu' OR 'respiratory virus' $O R$ 'respiratory infection' OR 'respiratory tract infection' \#3: \#1 AND \#2.

The search results were surveyed for methodological articles. Review articles were excluded, but the reference lists in all retrieved review papers were searched for additional related articles. In addition, a manual search was performed with the corresponding authors' reference database.

\section{Selection}

Two authors (B.J.C. and Y.Z.) independently evaluated the titles and abstracts of all studies for potential inclusion in this review. The same authors then reviewed full-length versions of selected articles to determine inclusion. When consensus was not reached, discussion and further study evaluation with other authors was used to resolve data extraction discrepancies. Articles were included in the review if they (1) described controlled volunteer studies of influenza virus filtration of face masks or respirators,

(2) described observational or intervention studies of face masks or respirators to prevent influenza or influenza-like illness (ILI) in healthcare settings, (3) described observational or intervention studies of face masks or respirators to prevent influenza or ILI in community settings. Studies focused on specific non-influenza respiratory infections, such as SARS, were excluded. The initial search resulted in 279 citations. Fifty-six articles were accepted at the abstract stage and finally 12 articles were considered relevant for inclusion in this review (Fig. 1).

\section{RESULTS}

\section{Experimental volunteer studies}

We identified one study that examined the efficacy of face masks in filtering influenza virus in volunteer subjects. Johnson and colleagues tested the performance of surgical and N95 masks to filter virus in nine volunteers with confirmed influenza A or B virus infection [7]. Participants coughed five times onto a Petri dish containing viral transport medium held

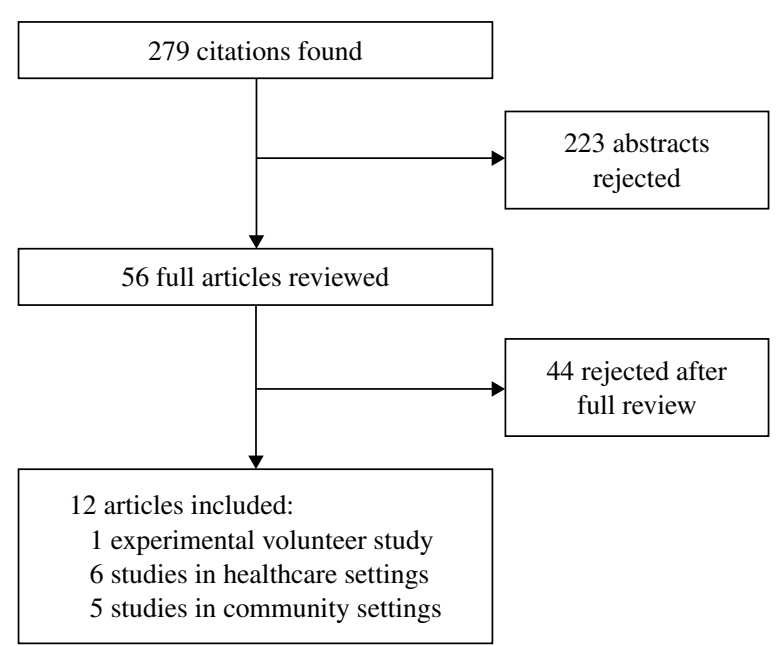

Fig. 1. Flow diagram of the process and results of article selection.

$20 \mathrm{~cm}$ in front of their mouth. The experiment was repeated with subjects wearing a surgical mask, and wearing an N95 respirator. While influenza virus could be detected by RT-PCR in all nine volunteers without a mask, no influenza virus could be detected on the Petri dish specimens when participants wore either type of face mask. A limitation was that the study did not consider the role of leakage around the sides of the mask.

\section{Studies in healthcare settings}

We identified six studies of face mask use in healthcare settings (Table 1) [8-13]. Because the study designs, participants, interventions and reported outcome measures varied markedly, we focused on describing the studies, their results, their applicability and their limitations and on qualitative synthesis rather than meta-analysis.

A randomized controlled trial in Canada found no significant differences in protection against laboratoryconfirmed influenza infection associated with the use of surgical masks or N95 masks among nurses [absolute risk difference $-0.73 \%, 95 \%$ confidence interval (CI) -8.8 to $7 \cdot 3$ ] with $24 \%$ of nurses in the surgical mask arm having laboratory-confirmed infection during an influenza season [8].

A randomized controlled trial in Japan allocated 32 healthcare personnel to wearing surgical face masks or not, but was underpowered to detect significant differences between arms with one observed acute respiratory illness in each arm of the study during the follow-up period [9]. 
Table 1. Studies conducted in healthcare settings

\begin{tabular}{|c|c|c|c|c|c|c|}
\hline Study & Setting & $\begin{array}{l}\text { Participants and } \\
\text { follow-up }\end{array}$ & Study design & Interventions evaluated & Main outcomes & Findings \\
\hline $\begin{array}{l}\text { Loeb } \\
\text { et al. [8] }\end{array}$ & $\begin{array}{l}8 \text { tertiary-care hospitals, } \\
\text { Ontario, 2008-2009 }\end{array}$ & 446 nurses & RCT & $\begin{array}{l}\text { N95 respirators, } \\
\text { surgical masks }\end{array}$ & $\begin{array}{l}\text { Seroconversion or } \\
\text { RT-PCR-confirmed } \\
\text { influenza infection }\end{array}$ & $\begin{array}{l}\text { No significant difference between } \\
\text { N95 and surgical masks }\end{array}$ \\
\hline $\begin{array}{l}\text { Jacobs } \\
\text { et al. }[9]\end{array}$ & $\begin{array}{l}\text { Tertiary-care hospital in } \\
\text { Tokyo, } 2008\end{array}$ & $\begin{array}{l}32 \text { individuals } \\
\text { followed for } 77 \text { days }\end{array}$ & $\mathrm{RCT}$ & Surgical masks, control & Self-reported colds & $\begin{array}{l}\text { No significant differences between } \\
\text { mask group and control group }\end{array}$ \\
\hline $\mathrm{Ng}$ et al. $[10]$ & $\begin{array}{l}\text { Teaching hospital in } \\
\text { Hong Kong, } 2007\end{array}$ & 133 healthcare workers & Cross-sectional & $\begin{array}{l}\text { Vaccination, use of } \\
\text { personal protective } \\
\text { equipment, hand } \\
\text { washing }\end{array}$ & $\begin{array}{l}\text { Self-reported } \\
\text { influenza-like } \\
\text { illness }\end{array}$ & $\begin{array}{l}\text { Suboptimal use of standard } \\
\text { precautions during high-risk } \\
\text { procedures associated with higher } \\
\text { risk of infection }\end{array}$ \\
\hline $\begin{array}{l}\text { Al-Asmary } \\
\text { et al. }[11]\end{array}$ & $\begin{array}{l}\text { Medical personnel in two } \\
\text { Hajj mission hospitals, } \\
\text { Saudi Arabia, } 2004\end{array}$ & 250 medical personnel & Cross-sectional & $\begin{array}{l}\text { Vaccination, face } \\
\text { masks, hand hygiene }\end{array}$ & $\begin{array}{l}\text { Self-reported acute } \\
\text { respiratory illness }\end{array}$ & $\begin{array}{l}\text { No significant protective effect of } \\
\text { face masks }\end{array}$ \\
\hline $\begin{array}{l}\text { Davies } \\
\text { et al. [12] }\end{array}$ & $\begin{array}{l}\text { General practice and a } \\
\text { teaching hospital, } \\
\text { 1991-1992 }\end{array}$ & 50 dental surgeons & Cross-sectional & Masks and spectacles & Seropositivity & $\begin{array}{l}\text { No significant differences by } \\
\text { mask use }\end{array}$ \\
\hline $\begin{array}{l}\text { Hobday \& } \\
\text { Cason [13] }\end{array}$ & $\begin{array}{l}\text { 'Open air' hospital in } \\
\text { Boston, } 1918\end{array}$ & Patients and staff & Observational & $\begin{array}{l}\text { Ventilation, use of } \\
\text { personal protective } \\
\text { equipment, hand } \\
\text { washing }\end{array}$ & Mortality & $\begin{array}{l}\text { Low case-fatality rate could be } \\
\text { associated with use of natural } \\
\text { ventilation and gauze face masks }\end{array}$ \\
\hline
\end{tabular}

RCT, Randomized controlled trial. 


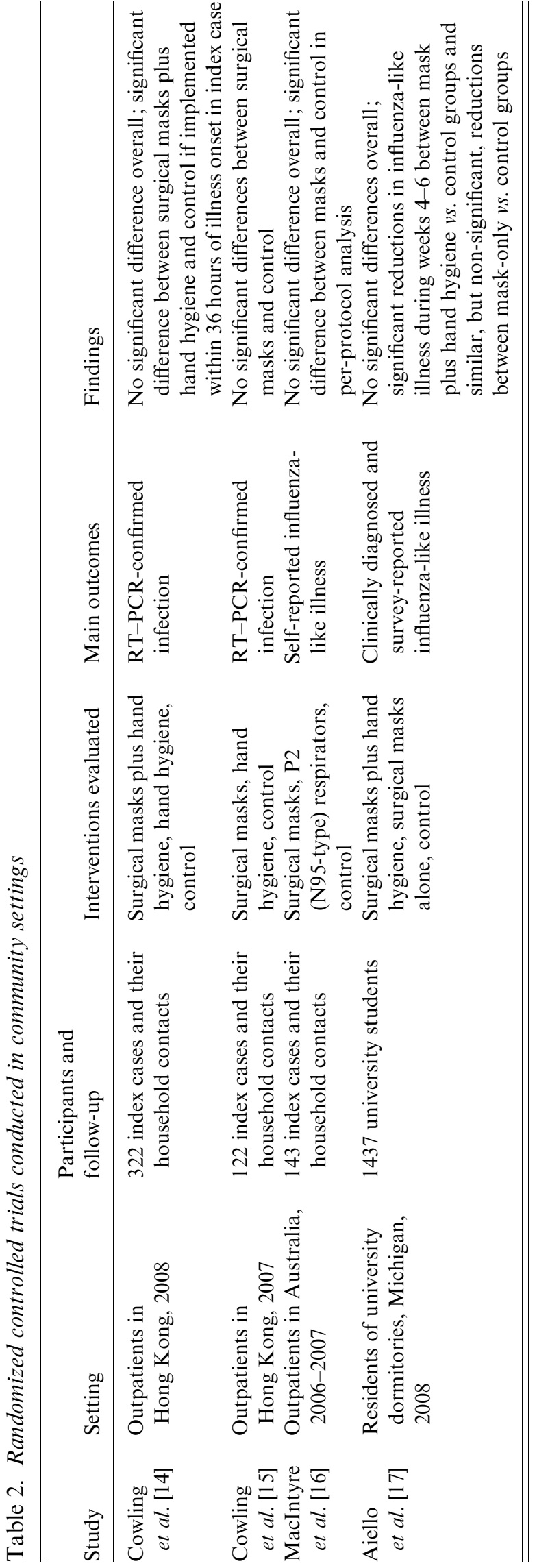

A survey of 133 nurses in Hong Kong found that suboptimal adherence to wearing a face shield during high-risk procedures [adjusted odds ratio (OR) 3.56, $95 \%$ CI $1 \cdot 18-10 \cdot 69$ ] was associated with higher risk of ILI, while suboptimal adherence to use of gloves and gowns were also associated with higher adjusted risk of ILI although not statistically significant [10]. Two other cross-sectional studies found no evidence for a protective effect of face masks against infection [11, 12]. Finally, Hobday \& Cason [13] speculated that natural ventilation, hand hygiene and gauze face masks were associated with fewer observed deaths in open-air hospitals in Boston during the 1918-1919 influenza A (H1N1) 'Spanish flu' pandemic, although there were many potential confounders.

\section{Studies in community settings}

We identified four randomized controlled trials that examined the effectiveness of face masks to prevent respiratory virus transmission in community settings [14-16] (Table 2). In a household-based study in Hong Kong, index cases and household members were randomized to three arms, including control, hand hygiene and hand hygiene plus surgical masks (to be worn by the index case and household members) [14]. In the primary intention-to-treat analysis there was no statistically significant difference in laboratory-confirmed influenza in household contacts across intervention groups. However when a prespecified analysis restricted attention to 154 households in which the intervention was applied within 36 hours of symptom onset in the index case, statistically significant reductions in laboratory-confirmed influenza virus infections in household contacts were observed in the face mask and hand hygiene arm (adjusted OR $0 \cdot 33,95 \%$ CI $0 \cdot 13-0 \cdot 87$ ). Adherence to the face mask intervention in index cases was moderate, but poorer in household contacts. The pilot study with a similar design was underpowered to identify significant differences between study arms [15].

Another recent study randomized 145 symptomatic index cases aged $0-15$ years from outpatient clinics and their household members to three arms: control, surgical masks (worn by household contacts only), or N95-type respirators (worn by household contacts only) without fit-testing [16]. There were no differences in ILI in household contacts across intervention arms. A secondary per-protocol analysis found that adherent use of N95 or surgical masks significantly 
reduced the risk for ILI in household contacts (hazard ratio $0 \cdot 26,95 \%$ CI $0 \cdot 09-0 \cdot 77$ ) compared to nonadherent mask use or allocation to the control arm.

Aiello and colleagues described a study in which 1437 university students were randomized by dormitory to three arms: control, surgical masks alone, and surgical masks plus hand hygiene [17]. Students were followed for 6 weeks during the influenza season and assessed for clinically diagnosed or survey-reported ILI. Compared with the control group, significant reductions in ILI were observed during weeks 4-6 in the mask and hand hygiene group ranging from $35 \%$ (95\% CI 9-53) to $51 \%$ (95\% CI 13-73), after adjusting for vaccination and other covariates; similar reductions, although not statistically significant, were observed in the mask-only group compared to the control group. Neither mask use and hand hygiene nor mask use alone was associated with significant reduction in ILI rate cumulatively; continued subject recruitment (larger sample size) after study start, increased participation in the intervention later in the study, a late, mild influenza season, and/or interruption of the intervention for 1 week by spring break may explain this finding. The study was underpowered to determine the relative contribution of the protective effects of masks compared to hand hygiene.

Finally, Lo and colleagues [18] investigated respiratory virus isolations in specimens collected primarily from in-patients and compared virus isolations in Hong Kong in 2003 with the preceding years. Declines in the number and proportions of virus isolations were attributed to population increases in hygienic measures and widespread use of face masks, as well as social distancing during the SARS epidemic. However, the study could not distinguish the relative contributions of each intervention.

\section{DISCUSSION}

Our review highlights the limited evidence base supporting the efficacy or effectiveness of face masks to reduce influenza virus transmission. An important concern when determining which public health interventions could be useful in mitigating local influenza virus epidemics, and which infection control procedures are necessary to prevent nosocomial transmission, is the mode of influenza virus transmission between people and in the environment. Physical barriers would be most effective in limiting shortdistance transmission by direct or indirect contact and large droplet spread, while more comprehensive precautions would be required to prevent infection at longer distances via airborne spread of small (nuclei) droplet particles [19]. In healthcare settings, stringent precautions are recommended to protect against pathogens that are transmitted by the airborne route, including the use of N95-type respirators (which require fit testing), other personal protective equipment including gowns, gloves, head covers and face shields, and isolation of patients in negativepressure rooms [19]. There remains considerable controversy over the relative importance of the alternative modes of transmission for influenza virus. In a recent review, Brankston and colleagues concluded that natural influenza transmission in human beings occurs generally over short distance rather than over long distance [20]. Based on the same evidence, Tellier had earlier concluded that aerosol transmission occurs at appreciable rates [21], and cited further evidence in an updated review [22]. Weber \& Stilianakis [23] found that contact, large droplet and small droplet (aerosol) transmission are all potentially important modes of transmission for influenza virus.

If airborne transmission were important, it would be less likely that surgical masks will lead to reductions in infectiousness or protection against infection, if worn by ill or uninfected people, respectively. The primary argument against airborne transmission is as much one of absence of evidence as evidence of absence. While there are documented examples of long-distance airborne transmission of other pathogens including varicella zoster virus and Mycobacterium tuberculosis, the literature contain few compelling examples of airborne transmission of influenza virus [20], and several reports of scenarios where airborne transmission did not occur [24-27]. Further indirect evidence such as the substantial benefit of hand hygiene to prevent influenza transmission [14] is suggestive of direct or indirect contact as one of the most important modes of transmission for influenza virus in some settings. Further observational or intervention studies conducted in different latitudes during different times of the year could help to elucidate the role of temperature and humidity in mediating modes of transmission [28].

We did not identify any experimental volunteer studies that investigated whether surgical masks or N95 respirators could protect against infection. We identified one experimental study of face mask performance which involved participants with confirmed influenza virus infection [7], and the results suggested that surgical masks may be able to reduce 
infectiousness. In future similar studies it would be important to consider the potential for leakage around the sides of the mask in addition to direct penetration of infectious viral particles through the mask, if the results are to have practical implications for reduction of transmission in community and other settings [29]. Further studies are needed to investigate how mask and respirator performance varies with temperature and humidity, or under working conditions when moisture in exhaled breath or sweat may build up in face masks and hinder filtration or fit [30].

Few studies have been conducted in healthcare settings, and there is limited evidence to support the effectiveness of either surgical masks or N95 respirators to protect healthcare personnel [8-13]. One recent large trial in nurses found no difference in effectiveness between surgical masks and N95 respirators, although the confidence intervals were wide enough to include moderate effect sizes [8]. Further, larger studies are needed to confirm the noninferiority of surgical masks. Guidance provided by the World Health Organization for protection of healthcare workers against pandemic influenza A (H1N1) virus infection recommends the use of standard and droplet precautions (including surgical masks or a face shield) during most patient interactions, while N95 or equivalent respirators are recommended for aerosol-generating procedures [31]. One concern over the use of face masks or respirators in healthcare settings is the potential for negative psychosocial impacts on patients and children in particular, especially in regions outside Asia where masks are not routinely worn [32]. Long-term use of N95-type respirators is likely to lead to physical discomfort [33], and has been associated with headaches [34]. Considerable resources might be required to make available N95 respirators and other protective equipment to large numbers of healthcare personnel through the course of influenza epidemics or pandemics. Finally, there are likely to be difficulties in ensuring compliance in healthcare workers [35]. Nevertheless personal protective equipment has led to major improvements in general infection control procedures in the hospital setting [36-38] and should not be discounted due to the lack of available data examining influenza virus outcomes.

Three controlled studies of face mask effectiveness in the community setting used case-ascertained designs, where ill index cases were recruited from outpatient clinics and households were followed up for 7-10 days to observe secondary transmission [14-16].
The Hong Kong study applied surgical face masks to index cases and their household contacts [14, 15], while the Australian study applied surgical masks or N95-type respirators to household contacts only [16]. Neither study provides conclusive evidence that face masks are effective in primary intention-to-treat analyses, although statistical power was limited. Adherence was moderate in both studies, and a perprotocol analysis of the Australian study suggests that masks could be effective in reducing risk of infection [16]. In the Hong Kong study, index cases not allocated to the face mask intervention reported use of face masks, indicating some degree of contamination of the intervention, while adherence was lower in household contacts and the results may primarily support the use of masks in ill members to reduce infectiousness $[14,15]$.

The effectiveness of face masks is probably impacted by compliance issues in both the healthcare and community setting [14, 15, 35]. Various studies show a lower level of compliance with face masks [14, 15 ] or find lower reported acceptability of face masks [39] compared to hand hygiene behaviours and other non-pharmaceutical interventions. However, these studies do not seek to explain the reduced compliance, nor do they measure levels of compliance in the midst of an outbreak of pandemic influenza. Future research endeavours should investigate the influence of cultural and sociobehavioural factors (e.g. fear, stigma, altruism) on levels of compliance during a pandemic. Use of face masks in the community was very common during the SARS epidemic in Hong Kong, but not in Singapore [40], and cultural differences could also affect compliance.

Pandemic guidance provided by the World Health Organization for community settings advises that masks may be worn although effectiveness is uncertain particularly in open spaces [41]. Other health agencies, such as the US Centers for Disease Control and Prevention, are not recommending masks in the community setting, with the exception of high-risk individuals who care for the sick or spend time in large crowds in areas affected by the pandemic [42]. Wearing masks incorrectly may increase the risk of transmission [41]. Further studies of face mask use are now underway, including some with prospective designs that follow cohorts of initially uninfected people. These studies will be particularly important in addressing compliance to and effectiveness associated with sustained use of face masks beyond the acute scenarios of existing studies [14-16]. While fewer 
resources are required to conduct studies with outcomes based on self-reported signs and symptoms of acute respiratory infection, future studies could include acute and convalescent serology or repeated collection of clinical specimens to provide results specific to influenza virus infection.

In conclusion there remains a substantial gap in the scientific literature on the effectiveness of face masks to reduce transmission of influenza virus infection. While there is some experimental evidence that masks should be able to reduce infectiousness under controlled conditions [7], there is less evidence on whether this translates to effectiveness in natural settings. There is little evidence to support the effectiveness of face masks to reduce the risk of infection. Current research has several limitations including underpowered samples, limited generalizability, narrow intervention targeting and inconsistent testing protocols, different laboratory methods, and case definitions. Further in-vivo studies of face masks in infectious individuals are warranted to determine the proportion of exhaled virus that is trapped by the mask. More detailed volunteer challenge and volunteer transmission studies could be designed to include both infectious and susceptible participants, to evaluate the efficacy of face masks both in reducing infectiousness and reducing susceptibility. However, such studies would require substantial resources, and contrived experiments may have limited generalizability to the natural setting. Large intervention studies in healthcare and community settings are likely to provide the best evidence of the effectiveness of face masks in reducing transmission in pandemic and inter-pandemic periods and are an urgent priority to guide pandemic preparedness for second and subsequent waves of pandemic influenza $\mathrm{A}(\mathrm{H} 1 \mathrm{~N} 1)$ and future pandemics.

\section{ACKNOWLEDGEMENTS}

We thank Lincoln Lau for technical assistance. This work was supported by the Area of Excellence Scheme of the Hong Kong University Grants Committee (grant no. AoE/M-12/06). The funding body was not involved in the collection, analysis and interpretation of data, the writing of the manuscript, or the decision to submit for publication.

\section{DECLARATION OF INTEREST}

None.

\section{REFERENCES}

1. Fraser C, et al. Pandemic potential of a strain of influenza A (H1N1): early findings. Science 2009; 324: 1557-1561.

2. Wilson N, Baker MG. The emerging influenza pandemic: estimating the case fatality ratio. Eurosurveillance 2009 ; 14: 1-4.

3. World Health Organization (WHO) Writing Group. Nonpharmaceutical interventions for pandemic influenza, international measures. Emerging Infectious Diseases 2006; 12: 81-87.

4. World Health Organization (WHO) Writing Group. Nonpharmaceutical interventions for pandemic influenza, national and community measures. Emerging Infectious Diseases 2006; 12: 88-94.

5. Jefferson $\mathbf{T}$, et al. Physical interventions to interrupt or reduce the spread of respiratory viruses: systematic review. British Medical Journal 2008; 336: 77-80.

6. Moher D, et al. Preferred reporting items for systematic reviews and meta-analyses: the PRISMA statement. British Medical Journal 2009; 339: b2535.

7. Johnson DF, et al. A quantitative assessment of the efficacy of surgical and N95 masks to filter influenza virus in patients with acute influenza infection. Clinical Infectious Diseases 2009; 49: 275-277.

8. Loeb M, et al. Surgical mask vs N95 respirator for preventing influenza among health care workers: a randomized trial. Journal of the American Medical Association 2009; 302: 1865-1871.

9. Jacobs JL, et $\boldsymbol{a l}$. Use of surgical face masks to reduce the incidence of the common cold among health care workers in Japan: a randomized controlled trial. American Journal of Infection Control 2009; 37: 417419.

10. Ng TC, et al. Preventing healthcare workers from acquiring influenza. Infection Control and Hospital Epidemiology 2009; 30: 292-295.

11. Al-Asmary S, et al. Acute respiratory tract infections among Hajj medical mission personnel, Saudi Arabia. International Journal of Infectious Diseases 2007; 11: 268-272.

12. Davies K, et al. Seroepidemiological study of respiratory virus infections among dental surgeons. British Dental Journal 1994; 176: 262-265.

13. Hobday RA, Cason JW. The open-air treatment of pandemic influenza. American Journal of Public Health 2009; 2 (Suppl.): S236-242.

14. Cowling BJ, et al. Facemasks and hand hygiene to prevent influenza transmission in households: a randomized trial. Annals of Internal Medicine 2009; 151: 437-446.

15. Cowling BJ, et al. Preliminary findings of a randomized trial of non-pharmaceutical interventions to prevent influenza transmission in households. PLoS One 2008; 3: e2101.

16. MacIntyre CR, et al. Face mask use and control of respiratory virus transmission in households. Emerging Infectious Diseases 2009; 15: 233-241. 
17. Aiello AE, et al. Mask use, hand hygiene and seasonal influenza-like illness among young adults: a randomized intervention trial. Journal of Infectious Diseases (in press).

18. Lo J, et al. Respiratory infections during SARS outbreak, Hong Kong, 2003. Emerging Infectious Diseases 2005; 11: 1738-1741.

19. Siegel JD, et al. Guideline for isolation precautions: preventing transmission of infectious agents in healthcare settings, June 2007. Atlanta, Georgia: Public Health Service, US Department of Health and Human Services, Centers for Disease Control and Prevention, 2007.

20. Brankston G, et al. Transmission of influenza A in human beings. Lancet Infectious Diseases 2007; 7: 257-265.

21. Tellier R. Review of aerosol transmission of influenza A virus. Emerging Infectious Diseases 2006; 12: 16571662.

22. Tellier R. Aerosol transmission of influenza A virus: a review of new studies. Journal of the Royal Society Interface 2009; 6 (Suppl. 6): S783-790.

23. Weber TP, Stilianakis NI. Inactivation of influenza A viruses in the environment and modes of transmission: a critical review. Journal of Infection 2008; 57 : 361-373.

24. Blumenfeld $\mathbf{H}$, et al. Studies on influenza in the pandemic of 1957-1958. I. An epidemiologic, clinical and serologic investigation of an intrahospital epidemic, with a note on vaccination efficacy. Journal of Clinical Investigation 1959; 38: 199.

25. Awofeso N, et al. Influenza outbreak in a correctional facility. Australian and New Zealand Journal of Public Health 2001; 25 : 443-446.

26. Morens D, Rash V. Lessons from a nursing home outbreak of influenza A. Infection Control and Hospital Epidemiology 1995; 16: 275.

27. Han K, et al. Lack of airborne transmission during outbreak of pandemic (H1N1) 2009 among tour group members, China, June 2009. Emerging Infectious Diseases 2009; 15: 1578-1581.

28. Lowen AC, et al. High temperature (30 degrees C) blocks aerosol but not contact transmission of influenza virus. Journal of Virology 2008; 82: 5650-5652.

29. Weiss MM, et al. Disrupting the transmission of influenza a: face masks and ultraviolet light as control measures. American Journal of Public Health 2007; 97 (Suppl. 1): S32-37.

30. Rengasamy A, Zhuang Z, Berryann R. Respiratory protection against bioaerosols: literature review and research needs. American Journal of Infection Control 2004; 32: 345-354.
31. World Health Organization. Infection prevention and control in health care for confirmed or suspected cases of pandemic (H1N1) 2009 and influenza-like illnesses. Interim guidance (25 June 2009). 2009. (http:// www.who.int/csr/resources/publications/20090429_ infection_control_en.pdf). Accessed 11 August 2009.

32. Beck M, et al. Wearing masks in a pediatric hospital: developing practical guidelines. Canadian Journal of Public Health 2004; 95: 256-257.

33. Li Y, et al. Effects of wearing N95 and surgical facemasks on heart rate, thermal stress and subjective sensations. International Archives of Occupational and Environmental Health 2005; 78: 501-509.

34. Lim EC, et al. Headaches and the N95 face-mask amongst healthcare providers. Acta Neurologica Scandinavica 2006; 113: 199-202.

35. Seale $\mathbf{H}$, et al. A review of medical masks and respirators for use during an influenza pandemic. Influenza and Other Respiratory Viruses 2009; 3: 205-206.

36. Lu YT, et al. Viral load and outcome in SARS infection: the role of personal protective equipment in the emergency department. Journal of Emerging Medicine 2006; 30: 7-15.

37. Casanova L, et al. Methods for the recovery of a model virus from healthcare personal protective equipment. Journal of Applied Microbiology 2009; 106: 1244-1251.

38. Rutala WA, Weber DJ. A review of single-use and reusable gowns and drapes in health care. Infection Control and Hospital Epidemiology 2001; 22: 248-257.

39. Stebbins S, Downs JS, Vukotich Jr. CJ. Using nonpharmaceutical interventions to prevent influenza transmission in elementary school children: parent and teacher perspectives. Journal of Public Health Management and Practice 2009; 15: 112-117.

40. Leung GM, et al. A tale of two cities: community psychobehavioral surveillance and related impact on outbreak control in Hong Kong and Singapore during the severe acute respiratory syndrome epidemic. Infection Control and Hospital Epidemiology 2004; 25: 1033-1041.

41. World Health Organization. Advice on the use of masks in the community setting in Influenza A (H1N1) outbreaks. Interim guidance (3 May 2009), 2009. (http:// www. who.int/csr/resources/publications/Adviceuse maskscommunityrevised.pdf). Accessed 11 August 2009.

42. Centers for Disease Control and Prevention. Interim public health guidance for the use of facemasks and respirators in non-occupational community settings during an influenza pandemic, 2009. (http://www.pandemicflu. gov/plan/community/maskguidancecommunity.html). Accessed 30 August 2009. 